\title{
SHORT TERM WIND SPEED PREDICTION USING A NEW HYBRID MODEL WITH PASSIVE CONGREGATION
}

\author{
TarekAboueldahab \\ Cairo Metro Company \\ Ministry of Transport \\ Cairo, Egypt
}

\begin{abstract}
Short term wind speed predicting is essential in using wind energy as an alternative source of electrical power generation, thus the improvement of wind speed prediction accuracy becomes an important issue. Although many prediction models have been developed during the last few years, they suffer a poor performance becausetheir dependency on performing only the local search without the capability in performing the global search in the whole search space.To overcome this problem, we propose a new passive congregation term to the standard hybrid Genetic Algorithm / Particle Swarm Optimization (GA/ PSO) model in training Neural Network (NN) wind speed predictor. This term is based on the mutual cooperation between different particles in determining new positions rather than their selfish thinking. Experiment study shows significantly the influence of the passive congregation term in improving the performance accuracy compared to the standard model.
\end{abstract}

\section{General Terms}

Wind Speed Prediction,

\section{Key words}

Particle Swarm Optimization, Genetic Algorithm, Neural Networks, Passive Congregation.

\section{INTRODUCTION}

Wind energy is one of the lowest costs of electricity production among renewable energy sources, but is visible only as long as weather conditions allow, so to maintain economical dispatch of wind generation electricity, it is important to make short term predictions of future wind speed which directly affects generation capacity [1]. Without this ability, a wind farm operator is prone to allocate more generation units or supplemental energy reserves than necessary in order to ensure budgeted electricity outputs are met, with an end result of increased operating costs [2]. Thus, the further prediction accuracy improvement of the wind speed predication becomes a fundamental issue in the wind industry [2-3].

In short term wind speed prediction, Feedforward Neural Networks (FNN) trained using tabu search [4] and Recurrent Neural Networks (RNN) [5] have been used.Particle Swarm Optimization (PSO) which is a population based stochastic algorithm was used in training both FNN and RNN wind speed predictors to enhance their prediction accuracy [2]. Also, Genetic Algorithm (GA) operators was introduced to increase the training accuracy of PSO learning algorithm, to form GA/ PSO hybrid model to enable searching new regions in the search space [6-7].

However, in GA/ PSO model, the PSO algorithm is restricted in doing the local search around its best position and the global best position without any cooperation with other particles in the swarm while the GA operators are responsible in doing the global search by making particles fly to new regions in the search space leading to poor model performance in terms of accuracy, convergence speed and robustness [8]. To overcome this problem and improve the model performance, each particle in the swarm should be capable of performing the global search and not to be only restricted in local search [9]. Thus, we introduce our new proposed hybrid (GA/PSO) model with passive congregation inspired by the biological mechanisms of school of fishes and flock of birds based on inserting the position difference between other two particles into the particle position update equation. This term enables each particle to compare between its selfish thinking and cooperation with other particles to adjust its position in better regions the search space. We compare between the performance accuracy of our proposed hybrid passive congregation model and the standard one in short term wind speed prediction. Simulations results show significantly that the passive congregation term positively enhance the performance accuracy and keep the fast convergence speed and robustness. This paper is organized as follows: section 2 reviews main concepts of the hybrid (GA/PSO) model and section 3 introduces our proposed hybrid GA/PSO model with passive congregation. Experimental study is presented in section 4 and finally the conclusion and future work are presented in section 5 .

\section{HYBRID GA/PSO MODEL}

Particle Swarm Optimization (PSO) is a population based stochastic optimization technique developed by Kennedy and Eberhart[10]and each particle in the swarm represents a potential solution of the optimization problem in $D$ dimensional space. At the $k^{\text {th }}$ generation the $i^{\text {th }}$ particle in the swarm is represented by its current position $X_{i}(k)=$ $\left(x_{i 1}(k), x_{i 2}(k), \ldots, x_{i D}(k)\right)$, its current velocity $\mathrm{V}_{\mathrm{i}}(\mathrm{k})=$ $\left(\mathrm{v}_{\mathrm{i} 1}(\mathrm{k}), \mathrm{v}_{\mathrm{i} 2}(\mathrm{k}), \ldots, \mathrm{v}_{\mathrm{iD}}(\mathrm{k})\right)$, and its current fitness function $F_{i}(k)$, the particle position and velocity are updated in the next generation by the following equations

$$
\begin{gathered}
V_{i}(k+1)=w * V_{i}(k)+C_{1} * \varphi_{1} *\left(X_{g}-X_{i}(k)\right)+C_{2} * \varphi_{2} \\
*\left(X_{\text {ibest }}-X_{i}(k)\right)(1) \\
X_{i}(k+1)=X_{i}(k)+V_{i}(k+1)
\end{gathered}
$$

Also the $i^{t h}$ particle autographical memory remembering its best previous position $X_{\text {ibest }}(k)=\left(x_{\text {ibest } 1}(k), x_{\text {ibest } 2}(k), \ldots, x_{\text {ibestD }}(k)\right)$ associated with its current best fitness function $F_{b e s t}$ and $X_{g}$ is the published knowledge of the best current position found by all particles corresponding to the global best fitness function $F_{g}$. From equation (1), the first term represents the inertia of the particle pervious velocity and $w$ is the inertia weight; the second term is the social term representing the cooperation among all particles where $C_{1}$ is the social constants and the third term is the cognition term which represents the private thinking and the selfish behavior of the particle itself where 
$C_{2}$ is cognitive constants and $\operatorname{both} \varphi_{1}$ and $\varphi_{1}$ are random variables in the range $[0,1]$

The GA operators are used after the fitness evaluation of all the particles in each generation, the worst $\left(S^{*} \Psi\right)$ particles performance are discarded and removed from the population where $\Psi$ is the breeding ratio determining thediscarded proportion of the swarm of size Sand its value is arbitrary selected in the range $[0.0,1.0]$.From the remaining $\left(\mathrm{S}^{*}(1-\Psi)\right)$ particles, parents particles are selected randomly to undergo crossover operator producing new child particles using the Velocity Propelled Averaged Crossover (VPAC) to accelerate the new particles away from their parents' direction according to the following equation[6]:-

$X_{1}^{C}(k+1)=\frac{X_{1}(k)+X_{2}(k)}{2}-\beta_{1} * V_{1}(k) \quad(3-a)$

$X_{2}^{C}(k+1)=\frac{X_{1}(k)+X_{2}(k)}{2}-\beta_{1} * V_{2}(k) \quad(3-b)$

Where $X_{1}^{C}(k+1)$ and $X_{2}^{C}(k+1)$ are the positions of child 1 and 2 respectively, $X_{1}(k)$ and $X_{2}(k)$ are the positions of the parents particles and $V_{1}(k)$ and $V_{2}(k)$ are their associated velocities and $\beta_{1}$ and $\beta_{2}$ are two uniformly distributed random variables in the range $[0,1]$. For the mutation process, all variables in the new created child particles are equally probable to be mutated [7].

\section{PROPOSED HYBRID (GA/PSO) WITH PASSIVE CONGREGATION}

Biologists found out that in a spatially well-defined group such as school of fishes or flock of birds, each individual can monitor both the environment and its immediate surrounding such as the position and the speed of neighbors and proposed two types of biological mechanisms to realize these requirements which are active aggregation and passive congregation [11].

Active aggregation is the transfer of necessary and active information among different individuals in the entire group such as the place with the most food and displays their social behaviors, this mechanism can be represented by the social term in equation (1) because it transfers the best position found so far to all particles in the swarm. Passive congregation is an attraction from one individual to others but does not display social behaviors. Individuals may have law fidelity to other group members if they have little or no genetic relation to each others, also each individual has multitude of potential information from other group members helping in reducing the possibility of miss detection and incorrect interpretations helping individuals to make global search in the whole search space and find new regions with better solutions [11].

Based on passive congregation concept, the cognitive term in equation (1) is not enough to represent the passive congregation mechanism because it displays only its best previous position without thinking of other particles positions [10]. To fully represent this mechanism, other particles positions should be included using the passive congregation term $P_{i}(k+1)$ and can be written by the following equation

$P_{i}(k+1)=C_{3} * \varphi_{3} *\left(X_{l}(k)-X_{m}(k)\right)$

Where $C_{3}$ is the passive congregation constant and $\varphi_{3}$ is random variable in the range $[0,1]$ and $X_{l}(k)$ and $X_{\mathrm{m}}(\mathrm{k})$ are the positions of particles $l$ and $\mathrm{m}$ respectively. Thenew velocity associated with passive congregation $V_{i}^{P}(k+1)$ is constructed by inserting this passive congregation term instead of the cognitive term and is written as follows:-

$$
\begin{gathered}
V_{i}^{P}(k+1)=w * V_{i}(k)+C_{1} * \varphi_{1} *\left(X_{g}-X_{i}(k)\right) \\
+P_{i}(k+1)
\end{gathered}
$$

This velocity with passive congregation term is based on other cooperative thinking between different particles while the standard velocity in equation (2) is based on the selfish thinking of the particle without any cooperation with other particles. Consequently, the new position with this added passive congregation term $X_{i}^{P}(k+1)$ is calculated as follows:-

$X_{i}^{P}(k+1)=X_{i}(k)+V_{i}^{P}(k+1)$

The performance of the passive congregation term is compared with the performance of the cognitive term by comparing the new obtained fitness function $F_{i}^{P}(k+$ 1)associated with passive congregation term with the standard one $F_{i}(k+1)$ associated with the cognitive term. The best one of them is selected to be assigned to the particle fitnessand the new particle position and velocity are adjusted accordingly.

If the passive congregation term doesn't improve the model performance, so there is no genetic or little genetic relation between this particle and the other randomly selected particles. On the other hand if it yields better performance, so these particles help in making correct interpretation through the global search in the whole search space to find new position with better performance.Thus all particles in the swarm can perform the global search by comparing the model performance resulting from both the original cognitive term and the new added perturbation term.

The proposed algorithm can be summarized in the following steps

Stepl (Initialization): randomly create an initial swarm of particles and setup the required inertia, social, cognitive, and perturbation constants. At each generation, for each particle in the swarm do the following

Step2 (Cognitive term): update the velocity $V_{i}(k+1)$ and position $X_{i}(k+1)$ using the cognitive term according to equations (1-2) and compute its associated fitness function $F_{i}(k+1)$.

Step3 (Passive congregation term): randomly select two other particles to construct the position difference between themand calculate the passive congregation term $\mathrm{P}_{\mathrm{i}}(\mathrm{k}+1) \mathrm{using}$ equation (4) and compute the velocity $V_{i}^{P}(k+1)$ and position $X_{i}^{P}(k+1)$ using the passive congregation term according to equations (5-6) and compute its associated fitness function $F_{i}^{P}(k+1)$

Step4 (Fitness Check): Select the best one of these two fitness functions to be assigned to the particle fitness and modify accordingly both the local best and the global best

\section{Step6 (Genetic operators)}

Selection: select the particles with the worst performance part according to the breeding ratio $\Psi$ to be replaced with other particles selected randomly as parents particles from the remaining part of the swarm

Crossover: construct the new child particles $X^{C}(k)$ to replace the worst particles according to equations (3-a, 3-b). 
Mutation: select randomly with equal probability one variable in the search space from the new children particles to be mutated.

Return to step 2 to start a new generation.

\section{EXPERIMENTAL STUDY}

Our experiment will be carried out in short term wind speed prediction for the months of March, April, and May 2011, the data was obtained from TORONTO LESTER B. PEARSON INT'L A station in ONTARIO province in Canada and this data is found in the website (http://www.climate.weatheroffice.gc.ca/climateData) [12] and The different values selected for all the parameters used in our experiment is shown in Table 1

Table 1:- Values of Parameters used in the experimental study

\begin{tabular}{|l|l|}
\hline Parameter & Value \\
\hline Generation number & 100 \\
Swarm size & 50 \\
inertia weight & 1 \\
social constant & 2 \\
cognitive constant & 2 \\
passive congregation constant & 2 \\
breeding ration & 0.1 \\
\hline
\end{tabular}

The Input / Output representation which is also called Nonlinear Autoregressive Moving Average (NARMA) representation [13-14] of the actualwend speed can be represented by the following equation

$Y(n+T)=\varphi[Y(n), Y(n-T) \ldots, Y(n-D T)]$

Where $Y(n)$ is the actual wind speed at the $n^{\text {th }}$ sample.Tis the time interval and $D$ is the number of delayed samples, shown in Figures 1-3. and $\varphi(*)$ is the nonlinear dynamical mapping function of the actual wind speed.

Also, the NARMA representation of the predicted wind speed model can be represented by the following equation

$Y_{p}(n+T)=\hat{\varphi}\left[Y(n), Y(n-T), \ldots, Y(n-D T), X_{i}(k)\right]$

Where $Y_{p}(n+T)$ is the wind speed predictor output, $\hat{\varphi}(*)$ is the nonlinear dynamical approximation function of $\varphi(*)$, and $X_{i}(k)$ is the $i^{t h}$ particle at the $k^{t h}$ generation as described in section 2 and it represents the weight set of this approximation function.

The neural network architecture used as the wind speed time series predictor is chosen to be the Sigmoid Diagonal Recurrent Neural Network (SDRNN) because we have proved in our previous works that this architecture is better than other different architectures for reducing the error and increasing the accuracy in many applications [14-15]. Both the standard model and the passive congregation model are used to train the predictor weights set and theirprediction accuracy are compared to show the influence of our proposed model. The assessment of the prediction accuracy of reducing this fitness function as much as possible is done in terms of the maximum absolute difference (MAX), and the Mean Absolute Percentage Error (MAPE) and they are defined as follows [16 $-17]$ :

$M A X=\max _{n}\left(\left|Y(n)-Y_{p}(n)\right|\right)$

$M A P E=\frac{1}{N} \sum_{k=1}^{N}\left(\frac{\left|Y(n)-Y_{p}(n)\right|}{Y(n)} * 100\right)$

In our experiment, the time interval $T$ is set to be one hour while the number of the delayed samples $D$ is selected to be four samples. The hourly wind speed data are divided into two parts which are the training part and the testing part [2-3, 1617]; the first $80 \%$ of the data will be taken as a training data while theremaining $20 \%$ will be the testing data. The actual and predicted wind speed using our proposed passive congregation model

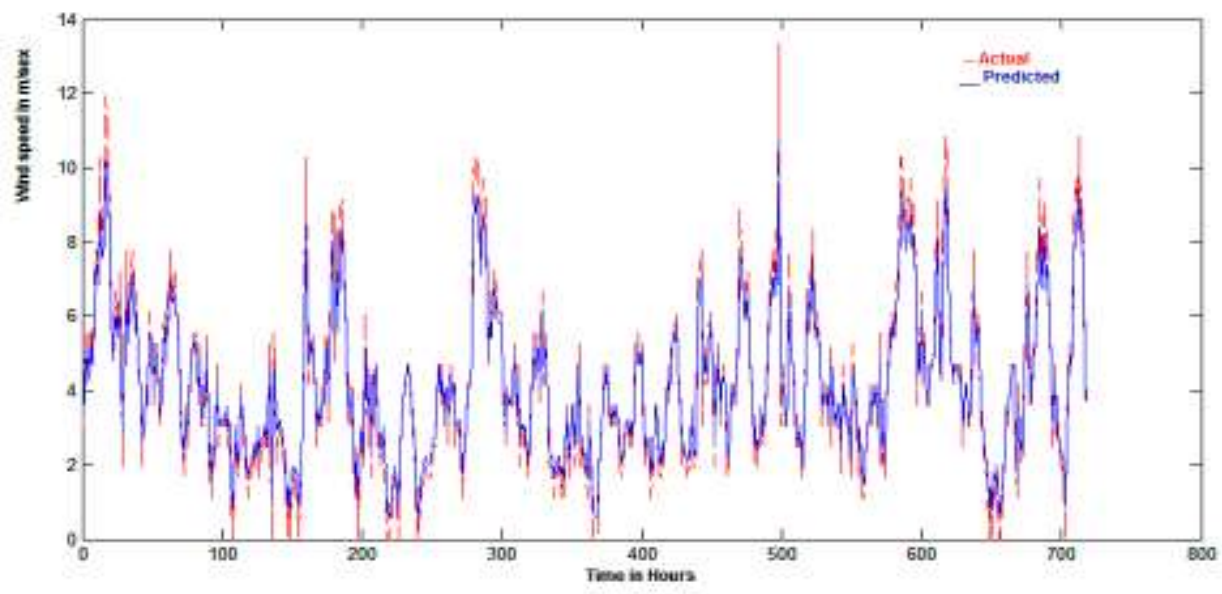

Figure 1: Wind speed in June 2012 


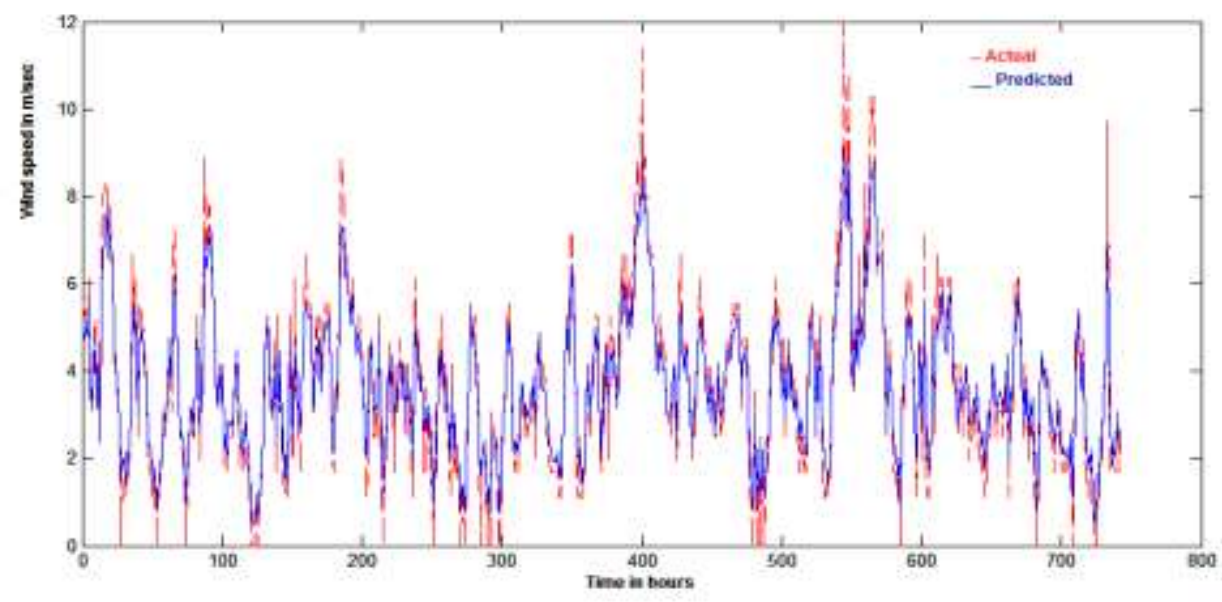

Figure2: Wind speed in July 2012

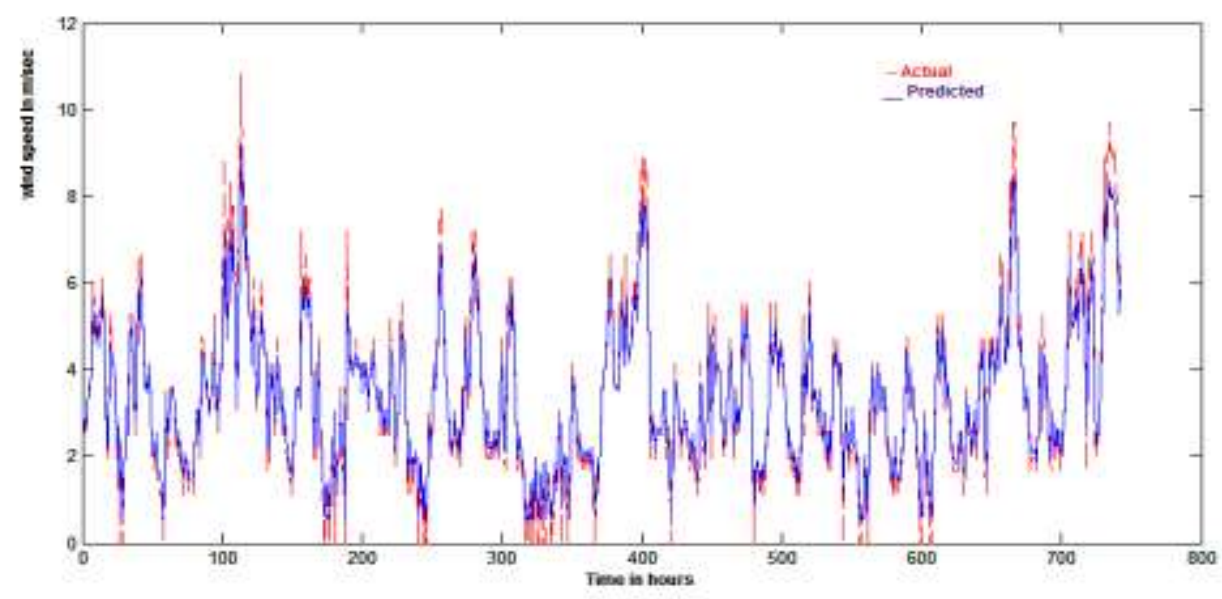

Figure3: Wind speed in August 2012

The fitness function $F_{p}(k)$ associated with the wind speed prediction is calculated as the sum square error between the actual and predicted as follows

$$
F_{p}(k)=\sum_{k=1}^{N}\left(Y(n)-Y_{p}(n)\right)^{2}
$$

The best fitness function obtained through all generations using both the standard model and passive congregation model for these three months are shown in Figures 4-6, 


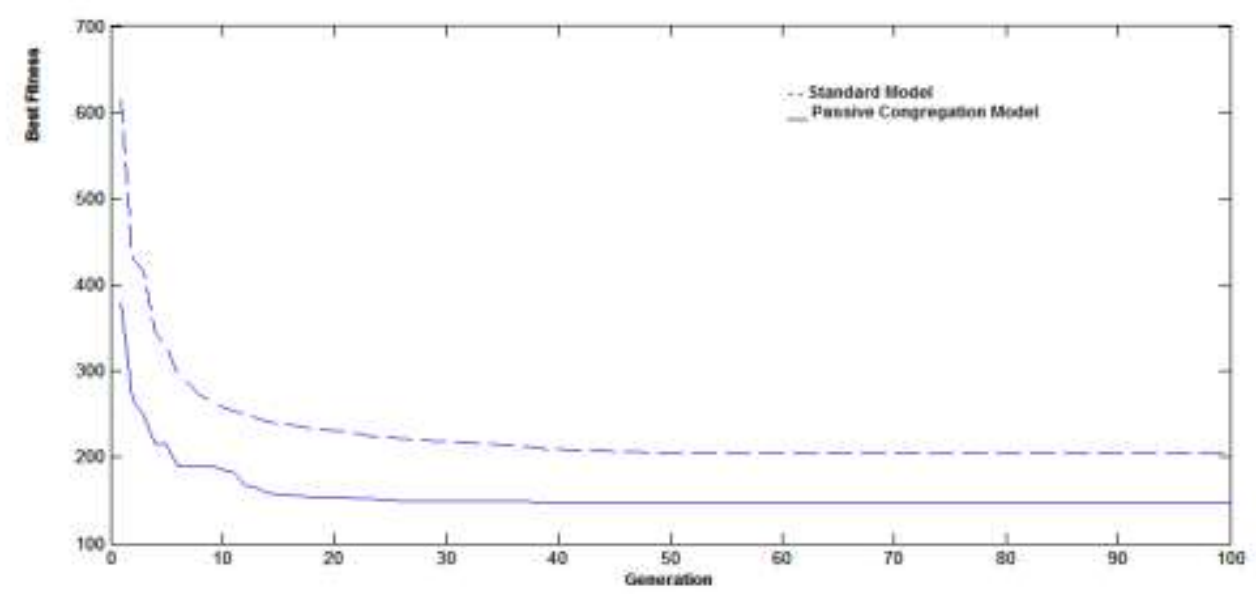

Figures 4:- Best Fitness function for the two models in June 2012

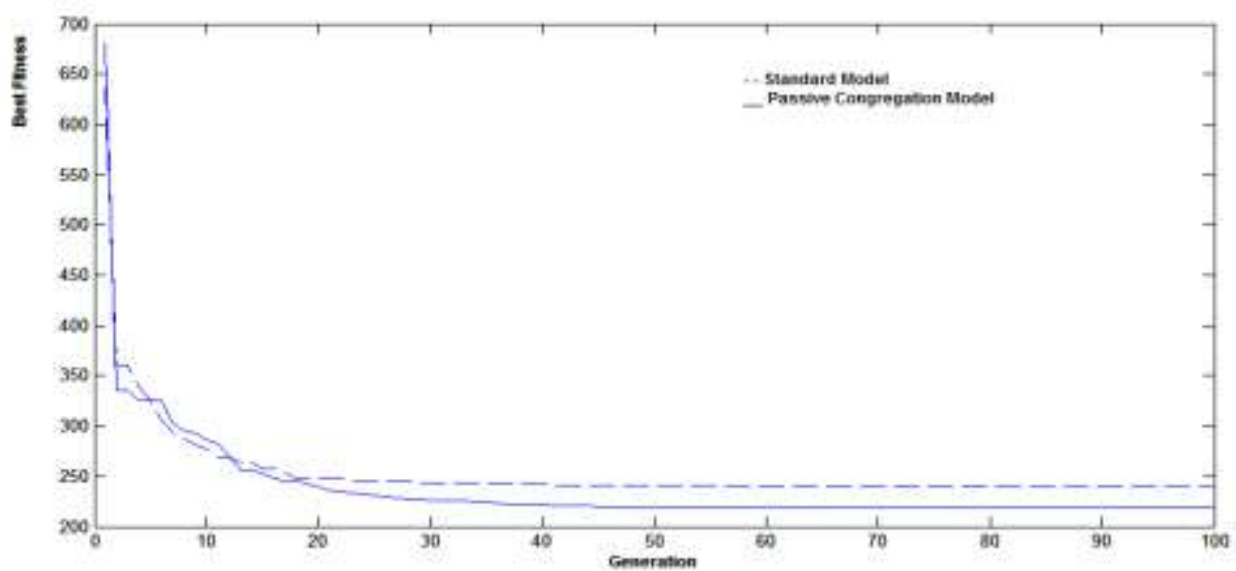

Figure 5:- Best Fitness function for the two models in July 2012

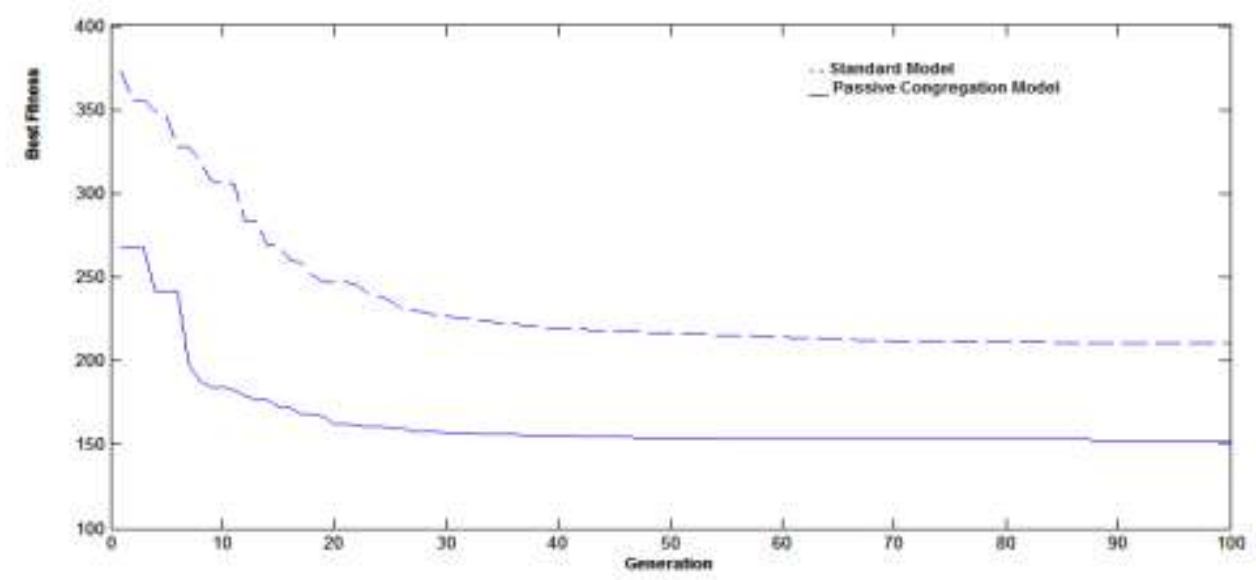

Figure 6:- Best Fitness function for the two models in August 2012

Theassessment of prediction accuracy using both models for the three months is shown in Table 2.

Table 2:- Performance measurements using standard model and passive congregation model 


\begin{tabular}{|c|c|c|c|c|c|c|}
\cline { 2 - 7 } \multicolumn{1}{c|}{} & \multicolumn{2}{c|}{ June } & \multicolumn{2}{c|}{ July } & \multicolumn{2}{c|}{ August } \\
\cline { 2 - 7 } \multicolumn{1}{c|}{} & $\begin{array}{c}\text { Stan } \\
\text { dard } \\
\text { mod } \\
\text { el }\end{array}$ & $\begin{array}{c}\text { Passiv } \\
\text { e } \\
\text { congre } \\
\text { gation } \\
\text { model }\end{array}$ & $\begin{array}{c}\text { Stan } \\
\text { dard } \\
\text { mod } \\
\text { el }\end{array}$ & $\begin{array}{c}\text { Passiv } \\
\text { e } \\
\text { congre } \\
\text { gation } \\
\text { model }\end{array}$ & $\begin{array}{c}\text { Stan } \\
\text { dard } \\
\text { mod } \\
\text { el }\end{array}$ & $\begin{array}{c}\text { Passiv } \\
\text { e } \\
\text { congre } \\
\text { gation } \\
\text { model }\end{array}$ \\
\hline M & 5.8 & 2.5 & 2.6 & 2.1 & 5.1 & 2.8 \\
AX & 14.5 & $12.6 \%$ & 15.9 & $12.7 \%$ & 18.2 & $15.4 \%$ \\
$\%$ & & & & & \\
\hline $\begin{array}{c}\text { MP } \\
\text { E }\end{array}$ & & & & & & \\
\hline
\end{tabular}

The plots in Figures4-6 show that our proposed model reduces successfully the required fitness function within a small number of generations while the standard one failed throughout all the generations, also the performance measurements shown in Table 2 indicate obviously that our proposed model yields better perditionaccuracy. This improvement is due to the existence of the passive congregation term which enables all particles to perform the global search discovering new regions with better fitness function in the whole search space while the poor performance of standard model is due to that the particles positions are dependent on their own thinking without knowing any information about other particles positions. Also, the mutual cooperation between different particles in finding new regions introduced by adding this passive congregation term is useful in avoiding miss detection and in increasing the correct interpretation.

\section{CONCLUSION AND FUTURE WORK}

A new hybrid (GA/PSO) model with passive congregationterm is presented to increase the prediction accuracy for short term wind speed prediction by enabling all particles in the swarm to perform the global search in the whole search space while only particles subjected to GA operators in the standard model are capable in performing this global search.

For the future work, our proposed passive congregation model can be applied in wind speed prediction using metrological data;also other time intervals varying from short term to long term will be used.

\section{REFERENCES}

[1] Potter C. W., and NegnevitskyM., "Very short-term wind forecasting for Tasmanian power generation," IEEE Trans. Power Syst., vol. 21, no. 2, pp. 965-972, May 2006.

[2] Richard L. Welch, Stephen M. Ruffing, and Ganesh K. Venayagamoorthy, "Comparison of feedforward and feedback neural network architectures for short term wind speed prediction" International Joint Conference on Neural Networks (IJCNN)pp. 3335-3340, 2009

[3] Andrew Kusiak, HaiyangZheng, and Zhe Song "ShortTerm Prediction of Wind Farm Power: A Data Mining Approach". IEEE Transactions on Energy Conversion, Vol.24, No. 1, pp 125-136,2009
[4] Han Shuang; Liu Yongqian; and Yang Yongping, "Taboo Search Algorithm Based ANN Model for Wind Speed Prediction " $2^{\text {nd }}$ international conference on Industrial Electronics and Applications, pp. 25992602,May 2007

[5] Senjyu T., Yona A., Urasaki N., and Funabashi T. "Application of recurrent neural Network to long-termahead generating power forecasting for wind power generator." Power Systems Conference and Exposition, pp. 1260-1265. IEEE Press, Atlanta, 2006

[6]Settles M., Nathan P., and Soule T., "Breeding Swarms: A New Approach to Recurrent Neural Network Training" In Proceeding of the Genetic and Evolutionary Computation Conference (GECCO-2005), pp. 185- 192, 2005.

[7] Ahmed A.A.E., GerminoL.T., and Antonio C.Z., "A Hybrid Particle Swarm Optimization Applied to Loss Power Minimization" IEEE Transactions on Power Systems, Vol. 20, No. 2, pp. 859-866, May 2005.

[8] VrugtJ.A., Robinso B.A., and Hyman J.M., "SelfAdaptive Multimethod Search for Global Optimization in Real-Parameter Space'IEEE Transactions on Evolutionary Computation, Vol. 13, No. 2, pp. $243-$ 259,APRIL 2009

[9] Premalatha K., and Natarajan A.M., "Hybrid PSO and GA for Global Maximization" International Journal Open Problems Compt. Math., Vol. 2, No. 4,pp. 597 - 608, 2009.

[10] Kennedy J., and EberhartR.C.,"Particle swarm optimization" Proceedings of IEEE International Conference on Neural Networks, Piscataway, NJ, pp.1942-1948, 1995.

[11] Parrish J. K., and Hammer W. M. "Animal Groups in Three Dimensions" Cambridge: Cambridge University Press, 1997.

\section{[12] http://www.climate.weatheroffice.gc.ca/climateData}

[13]Narendra K. S. and Parthasarathy K., "Identification and Control of Dynamical Systems Using Neural Networks," IEEE Transactions on Neural Networks, Vol. 1, No. 1, 1990, pp. 4-27.

[14] Tarek A., and Mouhamed F., "Identification and Adaptive Control of Dynamic Nonlinear Systems Using Sigmoid Diagonal Recurrent Neural Network" Intelligent Control and Automation, Vol. 2, No. 3, pp. 57-62, August 2011

[15] Tarek A., and Mouhamed F., "Adaptive Control of Dynamic Nonlinear Systems Using Sigmoid Diagonal Recurrent Neural Network."IEEE International Conference on Systems, Man, and Cybernetics (SMC), pp. 4341-4345, Istanbul. 2010.

[16]ChaouachiA., KamlerM., Ichikawa R., Hayashi H., and NagasakaK., " Neural Network Ensemble-based Solar Power Generation Short-Term Forecasting" World Academy of Science, Engineering and Technology 54, 2009

[17] TarekAboueldahab "A New Hybrid Model with Passive Congregation for Stock Market IndicesPrediction" World Academy of Science, Engineering and Technology, 66, 2012. 


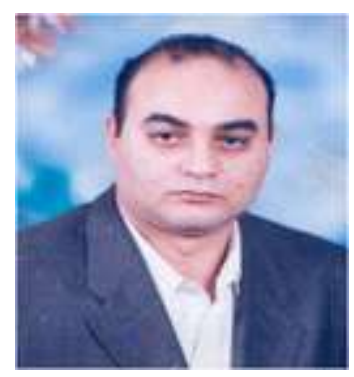

TarekAboueldahab: was born in April 1971 and obtained the bachelor degree in electronic and communication engineering from faculty of engineering-Cairo University in 1993 then a master degree in electronic andcommunication engineering -non linear control sectorfrom the same university in 1998. He is working inministry of transport, Cairo Metro Company since 1995and he is the manager of Research and development. His fields of interestinclude non-linear control, artificial intelligenceapplications, particle swarm optimization, and neuralnetworks

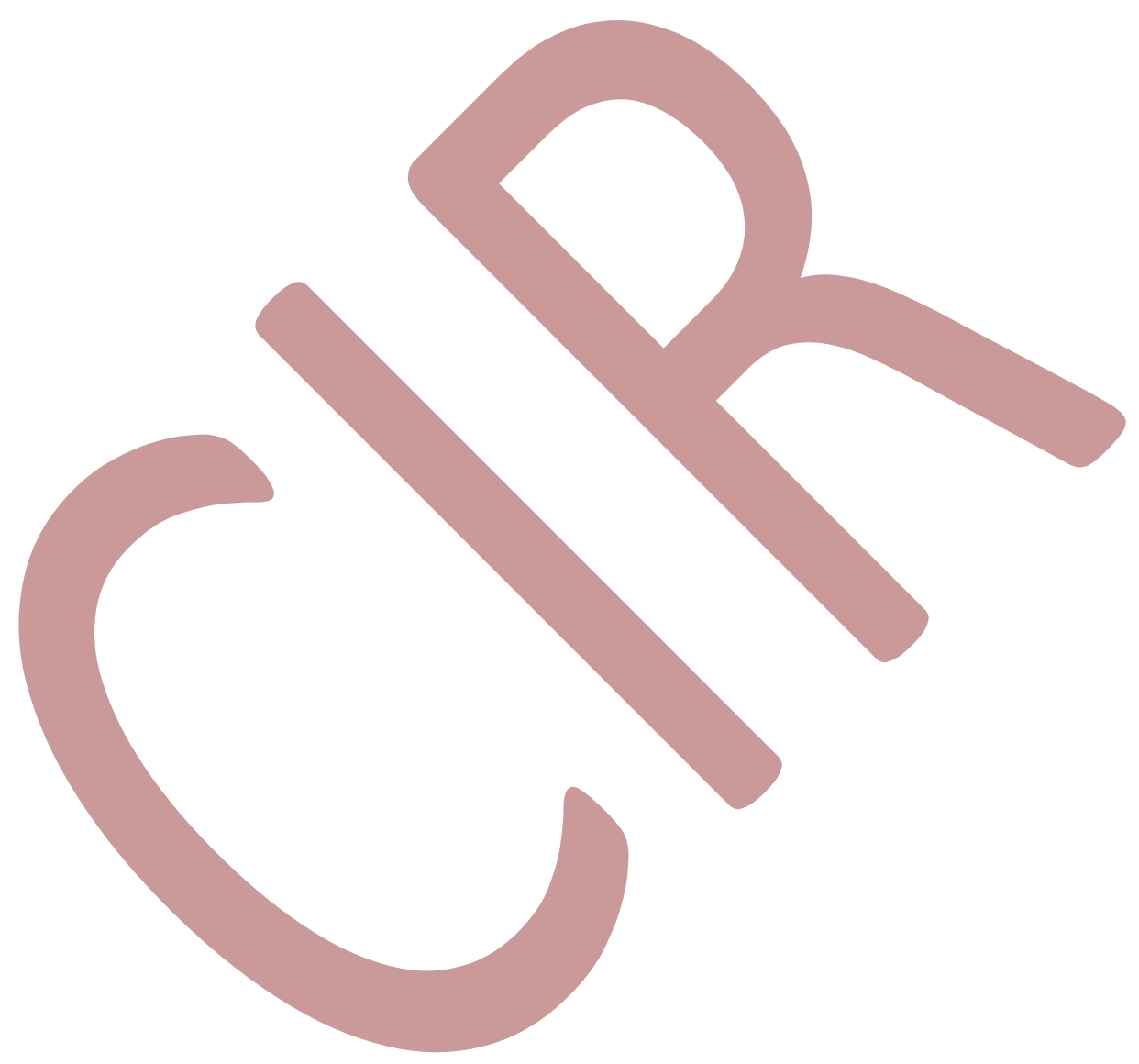

